\title{
The effect of lexical predictability on distributions of eye fixation durations
}

\author{
Adrian Staub \\ Published online: 17 December 2010 \\ (C) Psychonomic Society, Inc. 2010
}

\begin{abstract}
A word's predictability in context has a wellestablished effect on fixation durations in reading. To investigate how this effect is manifested in distributional terms, an experiment was carried out in which subjects read each of 50 target words twice, once in a high-predictability context and once in a low-predictability context. The exGaussian distribution was fit to each subject's first-fixation durations and single-fixation durations. For both measures, the $\mu$ parameter increased when a word was unpredictable, while the $\tau$ parameter was not significantly affected, indicating that a predictability manipulation shifts the distribution of fixation durations but does not affect the degree of skew. Vincentile plots showed that the mean exGaussian parameters described the typical distribution shapes extremely well. These results suggest that the predictability and frequency effects are functionally distinct, since a frequency manipulation has been shown to influence both $\mu$ and $\tau$. The results may also be seen as consistent with the finding from single-word recognition paradigms that semantic priming affects only $\mu$.
\end{abstract}

Keywords Distributional analysis · Visual word recognition $\cdot$ Eye movements in reading

The lexical variables that most reliably influence eye fixation durations in reading are a word's frequency (see, e.g., Inhoff \& Rayner, 1986; Rayner \& Duffy, 1986) and its predictability in context (e.g., Ehrlich \& Rayner, 1981; Rayner, Ashby, Pollatsek, \& Reichle, 2004), with longer fixations on low-frequency and unpredictable words. The E-Z Reader (Pollatsek, Reichle, \& Rayner, 2006) and

\footnotetext{
A. Staub $(\triangle)$

Department of Psychology, University of Massachusetts,

430 Tobin Hall,

Amherst, MA 01003, USA

e-mail: astaub@psych.umass.edu
}

SWIFT (Engbert, Nuthmann, Richter, \& Kliegl, 2005) models of eye movements in reading propose that fixation duration is an additive function of these variables, since experiments have not uncovered significant interactions between them (Altarriba, Kroll, Sholl, \& Rayner, 1996; Ashby, Rayner, \& Clifton, 2005; Rayner et al., 2004).

Recently, Staub, White, Drieghe, Hollway, and Rayner (2010) modeled how frequency affects individual subjects' distributions of fixation durations, using the ex-Gaussian distribution (Ratcliff, 1979). The ex-Gaussian is the convolution of a normal distribution with parameters $(\mu$, $\sigma)$ and an exponential distribution with single parameter $\tau$ (see Van Zandt, 2000, for other parameterizations of response time [RT] distributions). An experimental manipulation that affects mean RT by shifting the location of the distribution will influence primarily the $\mu$ parameter, while one that affects the mean by increasing the degree of skew will influence the $\tau$ parameter. A sizable literature has used the ex-Gaussian distribution to explore effects of a range of variables in single-word recognition tasks such as lexical decision, naming, and semantic categorization (e.g., Andrews \& Heathcote, 2001; Balota \& Spieler, 1999; Balota, Yap, Cortese, \& Watson, 2008; Plourde \& Besner, 1997; Yap \& Balota, 2007), but the Staub et al. study was the first to apply this method to eye movements in reading.

Staub et al.'s (2010) central conclusion was that, as in single-word tasks (Andrews \& Heathcote, 2001; Balota \& Spieler, 1999; Plourde \& Besner, 1997; Yap \& Balota, 2007), word frequency affects both shift and skew: The entire distribution of fixation durations is shifted to the right for low-frequency words, compared to high-frequency words, but the distribution for low-frequency words also has an especially pronounced right tail. In the data analyzed by Staub et al., the effects on the $\mu$ and $\tau$ parameters were approximately equal in size; in one data set (Drieghe, Rayner, \& Pollatsek, 2008), the frequency effect on the 
mean of the first-fixation duration measure (i.e., the duration of readers' first eye fixation on the critical word) was partitioned into a 16 -ms effect on $\mu$ and a $10-\mathrm{ms}$ effect on $\tau$; in a second data set (White, 2008), there was a 13-ms effect on $\mu$ and a 15 -ms effect on $\tau$.

The present article applies the same method to an investigation of the predictability effect. This is of interest for at least two reasons. First, the failure to obtain a significant Frequency $\mathrm{x}$ Predictability interaction effect suggests that these two variables may affect different stages or aspects of lexical processing; this contrasts with the view that they affect word recognition in similar manners-for example, by means of an influence on Bayesian probabilities (McDonald \& Shillcock, 2003). If the effects of the two variables are qualitatively distinct (e.g., with predictability influencing only the shift of the distribution or only skew), this would support the inference that the two effects are functionally distinct. Second, Balota et al. (2008) and Yap, Tse, and Balota (2009) have recently shown that a semantic priming manipulation shifts the distribution of RTs in speeded pronunciation and lexical decision tasks but does not affect skew (except when targets are degraded). It is of interest to know whether the predictability effect on fixation durations is similar, since it is an important question how this effect is related to semantic priming (Morris, 1994; see Lau, Phillips, \& Poeppel, 2008, for discussion with respect to the eventrelated potential literature).

A relatively large number of observations is required in order to obtain stable ex-Gaussian parameter estimates (Heathcote, Brown, \& Mewhort, 2002; Speckman \& Rouder, 2004). In the present study, each subject read 50 target words two times, once embedded in a sentence context that rendered the word highly predictable, and once in a context in which the word was unpredictable. Thus, it was possible to model the location and shape of the distributions of firstfixation durations and single-fixation durations (i.e., the subset of the first-fixation durations in which there were no additional fixations before leaving the word). ${ }^{1}$

\section{Method}

\section{Subjects}

Thirty-one students at the University of Massachusetts Amherst participated in the experiment in exchange for

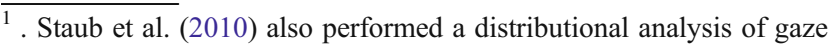
duration (the sum of all fixations before leaving a word), but noted that ex-Gaussian fitting is arguably less appropriate in this case, since the distribution of gaze durations is composed of a mixture of trials on which only a single first-pass fixation was made on the word and trials on which the word was refixated at least once.
}

course credit; 7 additional subjects were excluded due to poor calibration or excessive track loss. All subjects were native speakers of English, had normal or corrected-tonormal vision, and reported no reading or language-related disorders.

\section{Materials}

The materials for this experiment were based on those used by Altarriba et al. (1996), who presented 32 high-frequency and 32 low-frequency words in sentence frames in which the target word was either highly predictable (HP) or unpredictable (LP). (Unlike in the present experiment, each word was seen by each subject in only one of the two frames.) An example stimulus pair is shown in (1):

(1) a. The clown handed a big blue balloon to the little girl. (HP)

b. The boy selected a balloon from the bunch and released it. (LP)

Reexamination of the frequency statistics for the critical words revealed that they do not actually constitute separate high- and low-frequency classes. The distributions of frequencies for the two sets are nonoverlapping according to the Francis and Kučera (1982) norms used by Altarriba et al. (1996), but when the larger SUBTLex (Brysbaert \& New, 2009) or HAL (Burgess \& Livesay, 1998) corpora (available from the English Lexicon Project; Balota et al., 2002) are used, there is substantial overlap in the frequencies of the words in the two sets, such that they constitute a unimodal distribution.

New cloze norming was carried out for the sentence frames used by Altarriba et al. (1996). Each sentence fragment, up to but not including the critical word, was provided to between 37 and 42 UMass students, who were asked to provide the word that seemed most likely to come next in the sentence. Based on these norms, 14 of the original 64 sentence pairs were excluded, on the grounds that cloze probability was below .2 for the HP version ( 8 items), above .05 for the LP version (4 items), or both (2 items). This left 50 items for which the HP versions had a mean cloze probability of .60 and the LP versions had a mean cloze probability of .02 . The 50 target words had a mean SUBTLex frequency of 75.8 per million, ranging from cricket (2.8 per million) to money (641 per million). These words ranged from 4 to 10 characters in length, with a mean of 5.8. The 28 remaining sentences from Altarriba et al. were left in the experiment as fillers, although they were excluded from all analyses.

In the experiment, the sentences were presented in a pseudorandomized order, after eight practice trials. Each target word was read once in each half of the experiment, with half of the words first appearing in their HP sentence 
Table 1 Means on each eye movement measure, for highly predictable (HP) and unpredictable (LP) target words. Reading times are measured in milliseconds

\begin{tabular}{llllllrr}
\hline & Skipping & Prior Fix & First Fix & Single Fix & Gaze & Refixations & Regressions \\
\hline HP & $21.5 \%$ & 212.4 & 206.9 & 206.1 & 220.6 & $8.0 \%$ & $8.7 \%$ \\
LP & $14.6 \%$ & 213.3 & 222.4 & 225.4 & 243.0 & $9.3 \%$ & $13.6 \%$ \\
\hline
\end{tabular}

frame, and half in their LP sentence frame. Two-choice comprehension questions were presented after $1 / 3$ of trials; the subjects averaged $92.5 \%$ correct.

\section{Procedure}

Eye movements were recorded using an EyeLink 1000 (SR Research, Toronto, Ontario, Canada) eyetracker, interfaced with a PC computer, sampling at $1000 \mathrm{~Hz}$. Subjects were seated $55 \mathrm{~cm}$ from an Iiyama CRT monitor. The angular resolution of the eyetracker is 10-30 min of arc (less than one character). Viewing was binocular, but only the right eye was recorded. All sentences were displayed on a single line. Sentences were presented in 11-pt Monaco font. Before the experiment began, each subject was instructed to read for comprehension in a normal manner. A calibration procedure was then performed, and recalibration was carried out between trials as needed. The subject triggered the onset of each sentence by fixating a box on the left edge of the computer screen. The experiment lasted approximately $40 \mathrm{~min}$. The experiment was implemented using the EyeTrack software, and initial stages of data analysis were carried out using EyeDoctor and EyeDry (www.psych.umass.edu/eyelab/software/).

\section{Results}

Trials on which there was a track loss or any other data collection error $(5.5 \%$ of trials) were not analyzed. Fixations less than $80 \mathrm{~ms}$ in duration and within one character of the previous or the subsequent fixation were incorporated into this neighboring fixation. Remaining fixations shorter than $80 \mathrm{~ms}$ were deleted $(2.5 \%$ of fixations).

Data analysis focused on the readers' first inspection of the critical word in each sentence. Table 1 presents the firstfixation and single-fixation means, as well as several other measures. These include gaze duration (see footnote 1) and prior-fixation duration (i.e., the duration of the last fixation before fixating the critical word, used to assess any parafoveal-on-foveal effect; Kennedy \& Pynte, 2005; cf. Drieghe et al., 2008). Table 1 also includes the percentages of trials on which the words were skipped on first-pass reading; the percentages of trials on which, given that a word was fixated, it received more than one first-pass fixation; and the percentages of initial word inspections that ended with a regressive, rather than forward, saccade.

To assess the reliability of the reading time effects, a linear mixed effects model (Baayen, Davidson, \& Bates, 2008) was fit to the data for each dependent measure, with random intercepts for subjects and items, and with predictability as a fixed effect. Analysis of the binary dependent measures used mixed effects logistic regression, with the same random and fixed factors (Jaeger, 2008). Analyses were carried out using R, an open-source programming language and environment for statistical computing ( $\mathrm{R}$ Development Core Team, 2007), and in particular the lme4 package for linear mixed effects models (Bates, 2005; Bates \& Sarkar, 2007). The $p$ values for the linear mixed effects models are based on Markov chain Monte Carlo sampling (Bates, 2005); for logistic regression, $p$ values are based on the $z$ distribution. ${ }^{2}$

When unpredictable, a word received longer first-fixation durations $(b=17.34, S E=4.29, p<.001)$, single-fixation durations $(b=18.95, S E=4.68, p<.001)$, and gaze durations $(b=22.69, S E=5.70, p<.001)$. Unpredictable words were less likely to be skipped $(b=-0.51, S E=0.17$, $p<.01$ ), and were more likely to trigger regressive saccades $(b=0.58, S E=0.21, p<.01)$. The difference in the probability of refixation was not significant $(b=0.19, S E=$ $0.18, p=.28)$, nor was the difference in the duration of the prior fixation $(b=1.26, S E=4.34, p=.76)$. In sum, the predictability of the target word influenced the probability of skipping the word, the probability of regressing from the word, and all eye movement measures on the word itself, except the refixation probability.

Fitting of the ex-Gaussian distribution, for each subject in each condition, was carried out using the QMPE software (Cousineau, Brown, \& Heathcote, 2004; Heathcote et al., 2002). There were an average of 38.72 usable observations per subject and condition for first-fixation

\footnotetext{
2 . Larger models were constructed to assess the effects of log frequency, word length, the linear position of the target word in the sentence, and whether the word was being read for the first or second time. Only $\log$ frequency had a significant effect, showing significant or marginal effects on each of the reading time measures. The Frequency $\mathrm{x}$ Predictability interaction was not significant. See Slattery, Staub, and Rayner (2011) for an analysis of the frequency effect in this data set.
} 
Table 2 Means of the best-fitting ex-Gaussian parameters, for highly predictable (HP) and unpredictable (LP) conditions, for each measure, in milliseconds

\begin{tabular}{lrrrr}
\hline Variable & Mean & \multicolumn{1}{c}{$\mu$} & \multicolumn{1}{c}{$\sigma$} & \multicolumn{1}{c}{$\tau$} \\
\hline First-Fixation Duration & & & & \\
HP & 206.9 & 152.9 & 23.7 & 55.3 \\
LP & 222.4 & 167.0 & 28.3 & 58.3 \\
Predictability effect & 15.5 & 14.1 & 4.6 & 3.0 \\
Single-Fixation Duration & & & & \\
HP & 206.1 & 153.8 & 24.3 & 53.7 \\
LP & 225.4 & 167.9 & 29.0 & 59.2 \\
Predictability effect & 19.3 & 14.1 & 4.7 & 5.5 \\
\hline
\end{tabular}

duration, and an average of 35.37 for single-fixation duration. The same procedures were used as in Staub et al. (2010). All fits successfully converged. Table 2 provides the means of the $\mu, \sigma$, and $\tau$ parameters for each condition and each measure.

For first-fixation durations, predictability significantly affected the $\mu$ parameter, $t(30)=3.89, p<.001$. Predictability did not have a significant effect on $\sigma[t(30)=1.47, p=.15]$ or $\tau[t(30)=.53, p=.60]$. The same pattern held for singlefixation durations, with a significant predictability effect on $\mu[t(30)=3.64, p<.01]$ but not on $\sigma[t(30)=1.52, p=.14]$ or $\tau[t(30)=.89, p=.38]$. Thus, most of the effect of predictability on the means of the first-fixation and singlefixation measures was due to an effect on the location of the distribution of fixations, rather than to an effect on the skewness of the distribution. Only the effect on the location parameter, $\mu$, was statistically reliable.

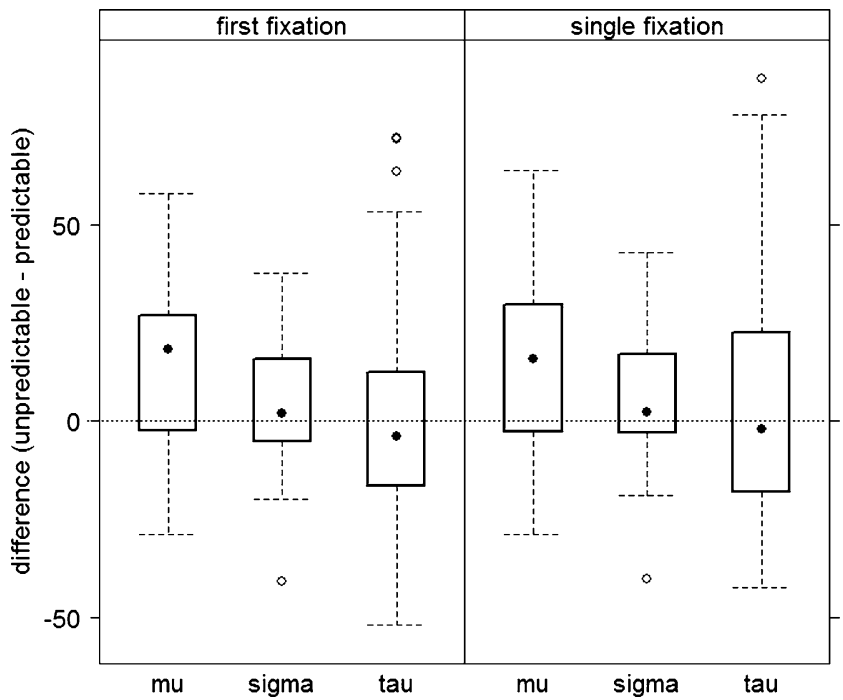

Fig. 1 Boxplots of subject differences between the unpredictable (LP) and highly predictable (HP) conditions for each of the three exGaussian parameters. (Positive values indicate increased parameter values in the LP condition.)
Figure 1 displays boxplots of subjects' difference scores between the LP and HP conditions, for each the three parameters. Most subjects showed increased $\mu$ in the LP condition; the median difference scores were 18.16 and $15.80 \mathrm{~ms}$ for first-fixation duration and single-fixation duration, respectively. However, the nonsignificant hint of a $\tau$ effect is actually driven by a few outliers for whom $\tau$ was much greater in the LP condition; the median $\tau$ difference scores were actually negative $(-3.95$ and $-2.00 \mathrm{~ms}$ ). Thus, examination of the distribution of effects across subjects strengthens the conclusion that only $\mu$ was reliably increased for LP words.

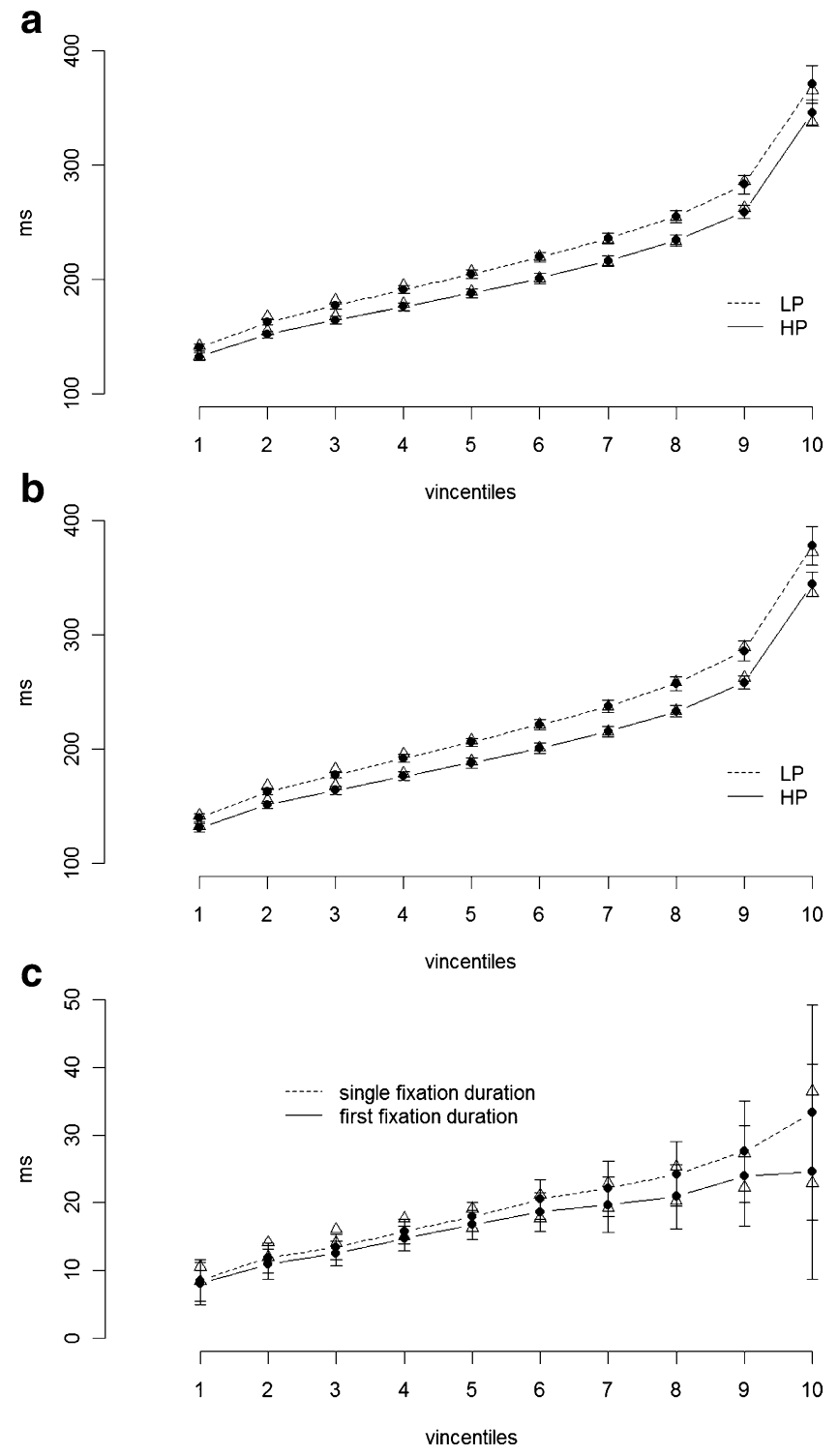

Fig. 2 Vincentile plots for first-fixation durations (a) and singlefixation durations (b), as a function of condition (unpredictable [LP] vs. highly predictable [HP]), and the mean differences between the LP and HP conditions (c), with standard errors. Predicted values based on the best-fitting ex-Gaussian parameters are represented by open triangles 
It is useful to confirm the results of ex-Gaussian analyses by means of a nonparametric method for assessing distribution shape. Figure 2 displays vincentile plots (Ratcliff, 1979; Vincent, 1912) of the data in each condition. These plots are constructed as follows. For each subject in each condition, the observations are divided into ten bins, such that the shortest $10 \%$ of the observations are in the first bin, the next shortest $10 \%$ in the next bin, and so on. The mean of the observations in each bin is calculated. The mean and standard error of these subject means is then plotted, with the first vincentile (the shortest durations) on the left and the tenth vincentile on the right. Figure $2 \mathrm{a}$ and $\mathrm{b}$ display these plots for first-fixation duration and singlefixation duration, respectively. Predicted vincentiles based on simulation from the ex-Gaussian parameters in Table 2 are represented on these plots with triangles. Note that these symbols are very close to (indeed, obscured by) the empirical vincentiles. Thus, the distribution generated by the mean ex-Gaussian parameters is very similar to the typical distribution in each condition. Finally, Figure 2c displays the difference between conditions in the empirical vincentiles. While this difference does increase from the fastest vincentiles to the slowest, especially for singlefixation duration, it is important to note that any increase is driven in large part by the few outlier subjects who did show a pronounced $\tau$ effect (see Fig. 1).

\section{Discussion}

The distribution of fixation durations is shifted to the right for unpredictable words, with little or no change in distributional shape. For both first-fixation and singlefixation durations, the $\mu$ parameter of the best-fitting exGaussian distribution was significantly increased for unpredictable words, but the $\tau$ parameter was not; the median effect on the $\tau$ parameter was actually negative. Vincentile plots demonstrated that the best-fitting parameters described the typical distribution in each condition extremely well.

This result may be seen as consistent with the finding of Balota et al. (2008) and Yap et al. (2009) that a semantic prime influences only $\mu$ in single-word recognition tasks. For both single-word RTs and fixation durations, word frequency affects both $\mu$ and $\tau$, but it appears that $\mu$ is the parameter primarily affected by manipulations that may affect lexical activation in advance of encountering a word. Taken together, these results argue against models of lexical processing according to which lexical frequency and contextual predictability have functionally identical roles (e.g., McDonald \& Shillcock, 2003).

Balota et al. (2008) interpreted the finding that a semantic prime influences only $\mu$ as reflecting a "head- start" to the word recognition process. Consistent with this conception is Matzke and Wagenmakers's (2009) finding that, within the Ratcliff diffusion model (Ratcliff \& McKoon, 2008), changes in the starting-point parameter (i.e., distance to a decision bound) affect $\mu$ but have only negligible effects on $\tau$. It remains to be seen how the metaphorical notion of a head start should be integrated into an explicit computational model of word recognition or, more specifically, eye movements in reading (e.g., Pollatsek et al., 2006). It is notable that the predictability of a word has a clear effect prior to direct fixation on the word, in the form of an effect on the skipping rate; the finding of a skipping effect in the present study is consistent with many previous studies (e.g., Ehrlich \& Rayner, 1981).

Author Note Thanks to Dan Hong and Victoria Neilsen for assistance with data collection. Thanks also to audiences at the 2010 CUNY Conference on Human Sentence Processing, New York, NY, and the First International Psycholinguistics Congress of ANPOLL, Rio de Janeiro, Brazil, for helpful discussion. The research presented here was supported in part by a Faculty Research Grant and a College of Social and Behavioral Sciences Matching Grant from the University of Massachusetts Amherst.

\section{References}

Altarriba, J., Kroll, J. F., Sholl, A., \& Rayner, K. (1996). The influence of lexical and conceptual constraints on reading mixedlanguage sentences: Evidence from eye fixations and naming times. Memory \& Cognition, 24, 477-492.

Andrews, S., \& Heathcote, A. (2001). Distinguishing common and task-specific processes in word identification: A matter of some moment? Journal of Experimental Psychology. Learning, Memory, and Cognition, 27, 514-544.

Ashby, J., Rayner, K., \& Clifton, C., Jr. (2005). Eye movements of highly skilled and average readers: Differential effects of frequency and predictability. The Quarterly Journal of Experimental Psychology, 58A, 1065-1086.

Baayen, R. H., Davidson, D. H., \& Bates, D. M. (2008). Mixed-effects modeling with crossed random effects for subjects and items. Journal of Memory and Language, 59, 390-412.

Balota, D. A., Cortese, M. J., Hutchison, K. A., Neely, J. H., Nelson, D., Simpson, G. B., \& Treiman, R. (2002). The english lexicon project: A web-based repository of descriptive and behavioral measures for 40,481 English words and nonwords. St. Louis, MO: Washington University. Available at http://elexicon.wustl.edu/

Balota, D. A., \& Spieler, D. H. (1999). Word frequency, repetition, and lexicality effects in word recognition tasks: Beyond measures of central tendency. Journal of Experimental Psychology: General, 128, 32-55.

Balota, D. A., Yap, M. J., Cortese, M. J., \& Watson, J. M. (2008). Beyond mean response latency: Response time distributional analyses of semantic priming. Journal of Memory and Language, $59,495-523$.

Bates, D. M. (2005). Fitting linear mixed models in R: Using the lme4 package. $R$ News: The Newsletter of the $R$ Project, 5(1), 27-30. 
Bates, D. M., \& Sarkar, D. (2007). Ime4: Linear mixed effects models using $S 4$ classes, $R$ package Version 0.998875-6. Madison, WI: Authors.

Brysbaert, M., \& New, B. (2009). Moving beyond Kučera and Francis: A critical evaluation of current word frequency norms and the introduction of a new and improved word frequency measure for American English. Behavior Research Methods, 41, 977-990.

Burgess, C., \& Livesay, K. (1998). The effect of corpus size in predicting reaction time in a basic word recognition task: Moving on from Kučera and Francis. Behavior Research Methods, Instruments, \& Computers, 30, 272-277.

Cousineau, D., Brown, S., \& Heathcote, A. (2004). Fitting distributions using maximum likelihood: Methods and packages. Behavior Research Methods, Instruments, \& Computers, 36, 742-756.

Drieghe, D., Rayner, K., \& Pollatsek, A. (2008). Mislocated fixations can account for parafoveal-on-foveal effects in eye movements during reading. The Quarterly Journal of Experimental Psychology, 61, 1239-1249.

Ehrlich, S. F., \& Rayner, K. (1981). Contextual effects on word perception and eye movements during reading. Journal of Verbal Learning and Verbal Behavior, 20, 641-655.

Engbert, R., Nuthmann, A., Richter, E. M., \& Kliegl, R. (2005). SWIFT: A dynamical model of saccade generation during reading. Psychological Review, 112, 777-813.

Francis, W. N., \& Kučera, H. (1982). Frequency analysis of English usage: Lexicon and grammar. Boston: Houghton Mifflin.

Heathcote, A., Brown, S., \& Mewhort, D. J. K. (2002). Quantile maximum likelihood estimation of response time distributions. Psychonomic Bulletin \& Review, 9, 394-401.

Inhoff, A. W., \& Rayner, K. (1986). Parafoveal word processing during eye fixations in reading: Effects of word frequency. Perception \& Psychophysics, 40, 431-439.

Jaeger, T. F. (2008). Categorical data analysis: Away from ANOVAs (transformation or not) and towards logit mixed models. Journal of Memory and Language, 59, 434-446.

Kennedy, A., \& Pynte, J. (2005). Parafoveal-on-foveal effects in normal reading. Vision Research, 45, 153-168.

Lau, E. F., Phillips, C., \& Poeppel, D. (2008). A cortical network for semantics: (De)constructing the N400. Nature Reviews. Neuroscience, 9, 920-933.

Matzke, D., \& Wagenmakers, E.-J. (2009). Psychological interpretation of the ex-Gaussian and shifted Wald parameters: A diffusion model analysis. Psychonomic Bulletin \& Review, 16, 798-817.

McDonald, S. A., \& Shillcock, R. C. (2003). Eye movements reveal the on-line computation of lexical probabilities during reading. Psychological Science, 14, 648-652.

Morris, R. K. (1994). Lexical and message-level sentence context effects on fixation times in reading. Journal of Experimental Psychology. Learning, Memory, and Cognition, 20, 92-103.
Plourde, C. E., \& Besner, D. (1997). On the locus of the word frequency effect in visual word recognition. Canadian Journal of Experimental Psychology, 51, 181-194.

Pollatsek, A., Reichle, E. D., \& Rayner, K. (2006). E-Z Reader: Testing the interface between cognition and eye movement control in reading. Cognitive Psychology, 52, 1-56.

Ratcliff, R. (1979). Group reaction time distributions and an analysis of distribution statistics. Psychological Bulletin, 86, 446-461.

Ratcliff, R., \& McKoon, G. (2008). The diffusion decision model: Theory and data for two-choice decision tasks. Neural Computation, 20, 873-922.

Rayner, K., Ashby, J., Pollatsek, A., \& Reichle, E. D. (2004). The effects of frequency and predictability on eye fixations in reading: Implications for the E-Z Reader model. Journal of Experimental Psychology: Human Perception and Performance, 30, 720-732.

Rayner, K., \& Duffy, S. (1986). Lexical complexity and fixation times in reading: Effects of word frequency, verb complexity, and lexical ambiguity. Memory \& Cognition, 14, 191-201.

R Development Core Team (2007). R: A language and environment for statistical computing. Vienna: R Foundation for Statistical Computing. Available from www.R-project.org

Slattery, T., Staub, A., \& Rayner, K. (2011). Word frequency and contextual predictability effects in reading: A comment on Hand, Miellet, O'Donnell, and Sereno (2010). Manuscript submitted for publication.

Speckman, P. L., \& Rouder, J. N. (2004). A comment on Heathcote, Brown, and Mewhort's QMLE method for response time distributions. Psychonomic Bulletin \& Review, 11, 574-576.

Staub, A., White, S. J., Drieghe, D., Hollway, E. C., \& Rayner, K. (2010). Distributional effects of word frequency on eye fixation durations. Journal of Experimental Psychology: Human Perception and Performance, 36, 1280-1293.

Van Zandt, T. (2000). How to fit a response time distribution. Psychonomic Bulletin \& Review, 7, 424-465.

Vincent, S. B. (1912). The function of the viborissae in the behavior of the white rat. Behavioral Monographs, 1(No. 5).

White, S. J. (2008). Eye movement control during reading: Effects of word frequency and orthographic familiarity. Journal of Experimental Psychology: Human Perception and Performance, 34, 205-223.

Yap, M. J., \& Balota, D. A. (2007). Additive and interactive effects on response time distributions in visual word recognition. Journal of Experimental Psychology. Learning, Memory, and Cognition, 33, 274-295.

Yap, M. J., Tse, C.-S., \& Balota, D. A. (2009). Individual differences in the joint effects of semantic priming and word frequency revealed by RT distributional analyses: The role of lexical integrity. Journal of Memory and Language, 61, 303-325. 